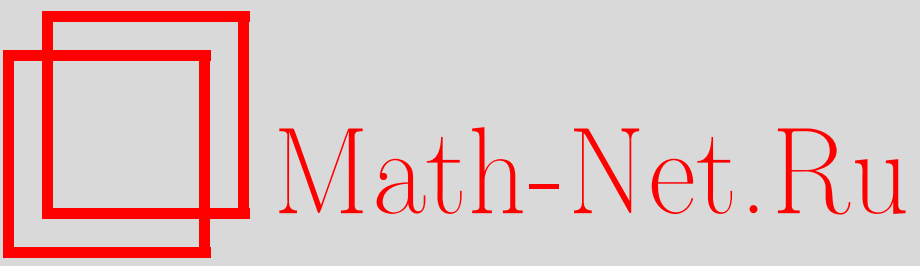

Б. С. Дарховский, Задача о неопределенной «разладке» случайной последовательности, Теория вероятн. и ее примен., 2011, том 56, выпуск 1, 30-46

DOI: https://doi.org/10.4213/tvp4322

Использование Общероссийского математического портала Math-Net.Ru подразумевает, что вы прочитали и согласны с пользовательским соглашением

http://www . mathnet.ru/rus/agreement

Параметры загрузки:

IP: 54.172 .240 .79

26 апреля 2023 г., 15:05:44 


\title{
ЗАДАЧА О НЕОПРЕДЕЛЕННОЙ «РАЗЛАДКЕ» СЛУЧАЙНОЙ ПОСЛЕДОВАТЕЛЬНОСТИ
}

\begin{abstract}
В статье рассматривается задача скорейшего обнаружения момента изменения распределения в последовательности независимых случайных величин (момента «разладки»). Предполагается, что априори известен конечный набор распределений последовательности, однако каким именно оно было до момента «разладки», а каким после - неизвестно. Таким образом, речь идет об обнаружении самого факта изменения без информации о его направлении (неопределенная «разладка»). Предлагается новый векторный критерий оценки качества метода обнаружения «разладки», который следует минимизировать. Для этого критерия получены неасимптотические априорные неравенства, дающие нижнюю границу качества. Предложен метод скорейшего обнаружения неопределенной «разладки», для которого асимптотически достигаются нижние границы введенного критерия.
\end{abstract}

Ключевые слова и фразыл: «разладка» случайной последовательности, асимптотически оптимальные последовательные тесты.

1. Введение. Проблема последовательного обнаружения момента изменения распределения случайной последовательности (как принято говорить, момента «разладки») уже в течение многих лет привлекает значительное внимание большого числа специалистов. Не останавливаясь на подробном анализе имеющихся в этой области работ, отсылаем читателя к тематическому выпуску журнала «Теория вероятностей и ее применения» (т. 53, в. 3, 2008) под редакцией А. Н. Ширяева (см. также статью [1]). Отметим также недавние работы [2]-[5], где рассматривалась задача обнаружения «разладки» и изолирования послеразладочного распределения.

В этой статье мы рассматриваем задачу о «разладке» в новой постановке: речь идет о скорейшем обнаружении момента изменения распределения случайной последовательности, но при этом априори неизвестно, какое именно распределение из заданного конечного списка было до момента «разладки», а какое - после. Предполагается, что известен конечный список плотностей распределений $f_{i}(x)$,

* Институт системного анализа РАН, пр-т 60-летия Октября, 9, 117312 Москва, В-312, Россия; e-mail: darbor2004@mail.ru 
$i \in I=\{1, \ldots, k\}$, которые могут соответствовать наблюдаемой последовательности, а «разладка» заключается в смене в неизвестный момент времени какой-либо $f_{i}(x)$ на какую-либо $f_{j}(x), j \neq i$. «Разладку» такого типа мы называем неопределенной (в дальнейшем, для краткости, мы будем использовать для такой неопределенной «разладки» обозначение $i \Rightarrow j)$.

Нам представляется, что подобная постановка может более адекватно отражать реальную ситуацию: точное знание распределений до и после «разладки» (вместо указания лишь списка, к которому они принадлежат) существенно сужает возможности приложений, так как получение подобной информации на практике достаточно сложно. Задача детектирования факта и момента изменения, на наш взгляд, не должна зависеть от априорной информации о том, в каком именно направлении произошла «разладка», нужно лишь знать общий список возможных распределений. Мы полагаем, что на первом этапе анализа важно как можно скорее «поймать» именно факт и момент изменения распределения, независимо от того, в каком направлении эта смена произошла, и лишь после детектирования момента «разладки» можно приступать к решению задачи о разделении выборки на два класса.

Насколько нам известно, подобным образом задача о «разладке» в литературе не ставилась даже для простых гипотез и независимых наблюдений.

Следуя нашим работам (см. [6] и имеющуюся там библиографию), мы вводим новый критерий качества детектирования момента «разладки». В данном случае это конечномерный вектор, компоненты которого представляют собой отношения (в подходящем масштабе) среднего времени от момента «разладки» до момента её обнаружения к среднему времени до первого ложного решения соответственно для всех возможных вариантов «разладки» $i \Rightarrow j,(i, j) \in I \times I$. Один из результатов работы состоит в установлении априорного неравенства, которому удовлетворяет критерий качества для широкого класса моментов остановки (м.о.). Кроме того, в работе предлагаются асимптотически оптимальные методы детектирования неопределенной «разладки».

Мы рассматриваем только последовательности независимых случайных величин, что позволяет существенно упростить изложение и облегчить для читателя восприятие идеи предлагаемого подхода. Результаты могут быть обобщены и на случай зависимых наблюдений.

Организация статьи такова. В п. 2 приведены предположения и формулируется задача, в п. 3 сформулировано априорное неравенство для произвольной компоненты критерия качества и приведено его доказательство. В п. 4 приводятся вспомогательные результаты, а в п. 5 указываются асимптотически оптимальные м.о. (т.е. такие м.о., для которых априорное неравенство переходит в равенство при стремлении к 
бесконечности «большого параметра» метода детектирования; заметим, что наличие подобного параметра характерно для всех разумных методов обнаружения). При этом в п. 5 рассмотрены случаи, когда список плотностей состоит из двух и трех функций. Переход к общему случаю конечного списка плотностей становится ясен из п. 5. В п. 6 приведена постановка упрощенного варианта задачи о неопределенной «разладке», когда происходит изменение только математического ожидания (которое может принимать конечное число значений). Здесь мы ограничиваемся кратким описанием (субоптимального) алгоритма детектирования для случая, когда математическое ожидание может принимать три различных значения.

2. Постановка задачи. Предположения. Пусть наблюдается последовательность независимых случайных величин (с.в.) $X=\left\{x_{n}\right\}_{n=1}^{\infty}$ такая, что плотность (для простоты, по мере Лебега) $\varphi_{n}(x)$ случайной величины $x_{n}$ имеет вид

$$
\varphi_{n}(x)= \begin{cases}f_{i}(x), & \text { если } 1 \leqslant n<m, \\ f_{j}(x), & \text { если } n \geqslant m,\end{cases}
$$

где $\mu\left\{x: f_{i}(x) \neq f_{j}(x)\right\}>0, i, j \in I, \mu-$ мера Лебега.

Момент «переключения» $m$ (априори неизвестный) мы называем моментом неопределенной разладки. Подчеркнем, что направление «переключения» (т.е. информачия о том, какая плотность была до «разладки», а какая - после) неизвестно, а известно лишь, что плотности принадлежат $\kappa$ конечному множеству функиий $\left\{f_{s}\right\}, s \in I$. В дальнейшем для краткости мы будем использовать $i \Rightarrow j$ для обозначения типа «переключения» в (1), а также употреблять термин «количество состояний» для обозначения числа элементов в множестве $I$.

Задача заключается в том, чтобы по наблюдениям скорейшим образом детектировать момент «разладки» (более точная постановка приводится ниже).

Всюду далее $\mathbf{P}_{m, i \Rightarrow j}$ и $\mathbf{E}_{m, i \Rightarrow j}$ обозначают меру и математическое ожидание, соответствующие последовательности $X$ с моментом «разладки» $m$ и направлением «переключения» $i \Rightarrow j$. Символы $\mathbf{P}_{\infty, i}$, $\mathbf{E}_{\infty, i}, i \in I$, соответствуют наблюдаемой последовательности без «разладки» с плотностью $f_{i}$. Мы также будем использовать обозначение $\mathbf{E}_{i} g(x)=\int g(x) f_{i}(x) d x, i \in I$.

Сформулируем предположения, при которых рассматривается задача.

(i) Для всех $(i, j) \in I \times I, i \neq j$, имеют место неравенства $0<\rho\left(f_{j}, f_{i}\right)<\infty$, где $\rho\left(f_{j}, f_{i}\right)=\int \ln \left(f_{j}(x) / f_{i}(x)\right) f_{j}(x) d x=$ $\mathbf{E}_{j} \ln \left(f_{j}(x) / f_{i}(x)\right)$ - расстояние Кульбака-Лейблера. 
(ii) Выполнено условие Крамера: для любой тройки $(i, j, k)$ $\mathbf{E}_{k}\left(f_{i}(x) / f_{j}(x)\right)^{t}<\infty$ при $|t| \leqslant H<\infty$.

(iii) Для любых $(i \neq j, k) \in I \times I$ таких, что $\mathbf{E}_{k} \ln \left(f_{i}(x) / f_{j}(x)\right)<0$, функция

$$
\varkappa_{i j k}(t)=\ln \int\left(\frac{f_{i}(x)}{f_{j}(x)}\right)^{t} f_{k}(x) d x
$$

имеет только два нуля: 0 и $t^{*}(i, j, k)>0$.

(iv) Для любых $(i \neq j, k) \in I \times I$ числа $\int \ln \left(f_{i}(x) / f_{j}(x)\right) f_{k}(x) d x$ отличны от нуля.

Теперь мы можем точно сформулировать задачу. Для определенности рассмотрим вариант «переключения» при «разладке» вида $i \Rightarrow j$. Предварительно заметим, что все разумные методы детектирования момента «разладки» (и соответствующие им м.о.) должны содержать некоторый «большой параметр» $C$, смысл которого в том, что с его увеличением вероятность ложного решения (т.е. остановки наблюдений в момент, когда «разладки» еще не было) стремится к нулю. Обычно роль такого параметра выполняет порог (например, в методе кумулятивных сумм (CUSUM)), но возможны и другие варианты.

Пусть $d(C, n)$ обозначает решающую функцию некоторого метода детектирования с «большим параметром» $C$ (т.е. измеримую по отношению к естественному потоку $\sigma$-алгебр функцию, порожденную наблюдениями и такую, что $d(C, n)=j(d(C, n)=i)$ соответствует решению о наличии (отсутствии) «разладки» рассматриваемого вида в момент времени $n)$. В частности, для метода CUSUM при обнаружении разладки вида $i \Rightarrow j$ решающая функция имеет вид

$$
\mathbb{D}_{i \Rightarrow j}(C, n)= \begin{cases}j, & \text { если } \max _{1 \leqslant s \leqslant n} \sum_{u=s}^{n} \ln \frac{f_{j}\left(x_{u}\right)}{f_{i}\left(x_{u}\right)}>C, \\ i \quad & \text { в противном случае. }\end{cases}
$$

Момент остановки наблюдений, соответствующий решающей функции $d(C, n)$, будем обозначать $\tau_{C}$, т.е. для разладки типа $i \Rightarrow j$ м.о. $\tau_{C}$ имеет вид $\tau_{C}=\inf \{n: d(C, n)=j\}$.

Определим теперь максимальную вероятность ложного решения (МВЛР) $\gamma_{i j}\left(\tau_{C}\right)$ для разладки типа $i \Rightarrow j$ и м.о. $\tau_{C}$, порожденного произвольной решающей функцией $d(C, n)$ :

$$
\gamma_{i j}\left(\tau_{C}\right) \stackrel{\text { def }}{=} \sup _{n} \mathbf{P}_{\infty, i}\{d(C, n)=j\} .
$$

З а м е ч а н и е. Величина МВЛР, вообще говоря, не совпадает с обычно используемой в задачах о «разладке» вероятностью ложной тревоги. Действительно, например, для стандартного метода CUSUM при 
обнаружении разладки вида $i \Rightarrow j$ и м.о. $\tau^{\mathrm{CS}}=\min \left\{n: \mathbb{D}_{i \Rightarrow j}(C, n)=j\right\}$ вероятность ложной тревоги $\beta_{i j}\left(\tau^{\mathrm{CS}}\right)$ выражается формулой

$$
\beta_{i j}\left(\tau^{\mathrm{CS}}\right) \stackrel{\text { def }}{=} \mathbf{P}_{\infty, i}\left\{\tau^{\mathrm{CS}}<\infty\right\}=\mathbf{P}_{\infty, i}\left\{\sup _{n} \max _{1 \leqslant s \leqslant n} \sum_{u=s}^{n} \ln \frac{f_{j}\left(x_{u}\right)}{f_{i}\left(x_{u}\right)}>C\right\}
$$

и, как нетрудно заметить, $\beta_{i j}\left(\tau^{\mathrm{CS}}\right) \geqslant \gamma_{i j}\left(\tau^{\mathrm{CS}}\right)$. Более того, из работы [9] можно извлечь, что (во всяком случае, в наших предположениях) вероятность ложной тревоги $\beta_{i j}\left(\tau^{\mathrm{CS}}\right)$ равна 1 при любом конечном значении положительного порога (именно с целью избежания этого в [9] предложено использовать растущий во времени порог). Поэтому использовать в качестве оперативной характеристики метода обнаружения вероятность ложной тревоги не всегда целесообразно (по крайней мере в тех случаях, когда рассматриваются м.о. с конечным математическим ожиданием при отсутствии «разладки»). В то же время введенная вероятность МВЛР прямо связана с важной оперативной характеристикой метода детектирования - средним временем до ложной тревоги (см. ниже лемму 1). Отметим также, что введение в рассмотрение процесса $d(C, n)$ может облегчить анализ конкретного метода детектирования.

Положим

$$
\alpha_{i j}\left(\tau_{C}\right) \stackrel{\text { def }}{=} \sup _{n} \mathbf{P}_{\infty, i}\left\{\tau_{C}=n\right\} .
$$

В силу включения $\left\{\tau_{C}=n\right\} \subset\{d(C, n)=j\}$ величина $\alpha_{i j}\left(\tau_{C}\right)$ является нижней гранищей для МВЛР.

Следуя нашим работам (см., например, [6]), мы предлагаем рассматривать для оценки качества метода детектирования типа $i \Rightarrow j$ (и соответствующего м.о. $\tau_{C}$ ) следующее отношение:

$$
\mathscr{K}_{i \Rightarrow j}\left(\tau_{C}, m\right) \stackrel{\text { def }}{=} \frac{\mathbf{E}_{m, i \Rightarrow j}\left(\tau_{C}-m \mid \tau_{C} \geqslant m\right)}{\left|\ln \gamma_{i j}\left(\tau_{C}\right)\right|}
$$

Для пояснения смысла введенного критерия качества приведем следующий результат (см. ссылку в [6]).

Лемма 1. Пусть случайная величина $\xi$ принимает положительнье челочисленнье значения и удовлетворяет условию $\sup _{n} \mathbf{P}\{\xi=n\} \leqslant a$. Тогда $\mathbf{E} \xi \geqslant(2 a)^{-1}(1+o(1))$ при а $\rightarrow 0$ и эта оченка точна.

Из этой леммы следует, что величина, стоящая в знаменателе формулы (2), эквивалентна логарифму среднего времени до первого ложного решения в наихудшем случае. В числителе (2) стоит условное среднее время запаздывания обнаружения (при условии, что момент обнаружения имел место после момента «разладки») для данного метода детектирования. Таким образом, для произвольного метода обнаружения критерий (2) представляет собой отношение (в подходящем масштабе, 
поскольку вероятность ложного решения обычно экспоненциально убывает к нулю) условного среднего времени запаздывания в обнаружении $\kappa$ наименьшему возможному среднему времени до первого ложного решения.

Нам представляется, что постановка задачи минимизации предложенного критерия в содержательном отношении ничуть не уступает распространенным постановкам, когда требуется минимизировать среднее время запаздывания при заданном ограничении на вероятность ложной тревоги или на среднее время до ложной тревоги.

3. Априорные неравенства. Зафиксируем два номера $i, j$ из списка $I$ и рассмотрим «разладку» типа $i \Rightarrow j$. Для произвольного $0<r<1$ определим положительное число $L\left(\tau_{C}, r, i\right)$ формулой

$$
L\left(\tau_{C}, r, i\right)=\min \left\{n: \sum_{k=n}^{\infty} \mathbf{P}_{\infty, i}\left(\tau_{C}=k\right) \leqslant r\right\}
$$

Определим следующий класс моментов остановки:

$$
\mathbb{M} \stackrel{\text { def }}{=}\left\{\tau_{C}: \mathbf{E}_{m, i \Rightarrow j}\left(\tau_{C}-m\right)^{+}<\infty \text { для любых фиксированных } i, j, C, m\right\}
$$

(здесь и далее $\left.a^{+}=\max (0, a)\right)$. Момент остановки $\tau_{C} \in \mathbb{M}$ мы называем невьлрожденньлм, если $0<\alpha_{i j}\left(\tau_{C}\right) \leqslant \gamma_{i j}\left(\tau_{C}\right)<1$.

Теорема 1. Пусть $\tau_{C}-$ невьрожденный м.о. из М. Пусть вылполненьл условия (i)-(iv). Тогда для любого фиксированного $m>0$ имеет место неравенство

$$
\begin{aligned}
\mathscr{K}_{i \Rightarrow j}\left(\tau_{C}, m\right) \geqslant & \frac{\rho^{-1}\left(f_{j}, f_{i}\right)}{\mathbf{P}_{\infty, i}\left(\tau_{C} \geqslant m\right)} \\
& \times\left[1-\frac{\ln \left(m+L\left(\tau_{C}, r^{*}, i\right)+1\right)}{\left|\ln \gamma_{i j}\left(\tau_{C}\right)\right|}-\frac{\rho\left(f_{j}, f_{i}\right)}{\left|\ln \gamma_{i j}\left(\tau_{C}\right)\right|}\right],
\end{aligned}
$$

где $r^{*}=\gamma_{i j}\left(\tau_{C}\right)$ (напомним, ито $\left.\rho\left(f_{j}, f_{i}\right)=\int \ln \left(f_{j}(x) / f_{i}(x)\right) f_{j}(x) d x\right)$.

Рассмотрим следующий класс м.о.:

$$
\mathfrak{M}=\left\{\tau_{C} \in \mathbb{M}: \limsup _{r \rightarrow 0} \frac{\ln L\left(\tau_{C}, r, i\right)}{|\ln r|}=0\right\} .
$$

В частности, к этому классу относятся м.о. с конечным (для всех конечных значений «большого параметра» $C$ ) математическим ожиданием и с экспоненциальными хвостами распределения при отсутствии «разладки». Известно (см. [9]), что при сделанных предположениях м.о. классического метода CUSUM обладает указанными свойствами.

Из теоремы 1 получаем такое следствие. 
Следствие. Пусть для м.о. $\tau_{C} \in \mathfrak{M}$ выполнено условие

$$
\limsup _{C \rightarrow \infty} \gamma_{i j}\left(\tau_{C}\right)=\limsup _{C \rightarrow \infty} \sup _{n} \mathbf{P}_{\infty, i}\{d(C, n)=j\}=0 .
$$

Тогда для любого фиксированного $m>0$

$$
\lim _{C \rightarrow \infty} \mathscr{K}_{i \Rightarrow j}\left(\tau_{C}, m\right)=\lim _{C \rightarrow \infty} \frac{\left.\mathbf{E}_{m, i \Rightarrow j} \tau_{C}-m \mid \tau_{C} \geqslant m\right)}{\left|\ln \gamma_{i j}\left(\tau_{C}\right)\right|} \geqslant \rho^{-1}\left(f_{j}, f_{i}\right) .
$$

Метод последовательного обнаружения «разладки» мы называем асимптотически оптимальным, если нижняя граница в этом неравенстве достигается.

Д ок аз а тель с тв о те о ре мы 1. Зафиксируем некоторое $m \geqslant 1$ и $i, j \in I$. Пусть $\tau_{C} \stackrel{\text { def }}{=} \tau \in \mathbb{M}-$ некоторый невырожденный м.о., $\gamma_{i j}\left(\tau_{C}\right) \stackrel{\text { def }}{=} \gamma-$ соответствующая максимальная вероятность ложного решения.

Определим число $L\left(\tau, r^{*}, i\right) \stackrel{\text { def }}{=} L(\gamma)$ из условия

$$
\sum_{k=L(\gamma)}^{\infty} \mathbf{P}_{\infty, i}(\tau=k) \leqslant \gamma
$$

Определим следующий м.о.: $\widetilde{\tau}=m+(\tau-m)^{+} \wedge L(\gamma)$. Очевидно, что $\widetilde{\tau}=m, m+1, \ldots, m+L(\gamma)$. В силу определения $\gamma$ и $\widetilde{\tau}$ имеем

$$
\begin{gathered}
\mathbf{P}_{\infty, i}(\widetilde{\tau}=m) \leqslant \sum_{s=1}^{m} \mathbf{P}_{\infty, i}(\tau=s) \\
\mathbf{P}_{\infty, i}(\widetilde{\tau}=m+L(\gamma)) \leqslant \sum_{s=m+L(\gamma)}^{\infty} \mathbf{P}_{\infty, i}(\tau=s) \leqslant \sum_{s=L(\gamma)}^{\infty} \mathbf{P}_{\infty, i}(\tau=s) \leqslant \gamma
\end{gathered}
$$

Далее, обозначая $X_{u}^{v}=\left\{x_{k}\right\}_{k=u}^{v}$, получаем

$$
\begin{aligned}
(m+L(\gamma)+1) \gamma & \geqslant \sum_{s=m}^{m+L(\gamma)} \mathbf{P}_{\infty, i}\{\tilde{\tau}=s\} \\
& =\sum_{s=m}^{m+L(\gamma)} \mathbf{E}_{m, i \Rightarrow j}\left(\mathbb{I}(\widetilde{\tau}=s) \frac{d \mathbf{P}_{\infty, i}}{d \mathbf{P}_{m, i \Rightarrow j}}\left(X_{1}^{s}\right)\right)
\end{aligned}
$$

(здесь и далее символ $\mathbb{I}(A)$ обозначает индикаторную функцию множества $A$ ). Так как наблюдаемая последовательность состоит из независимых случайных величин, то для $m+L(\gamma) \geqslant s \geqslant m$ имеем:

$$
\frac{d \mathbf{P}_{\infty, i}}{d \mathbf{P}_{m, i \Rightarrow j}}\left(X_{1}^{s}\right)=\prod_{k=m}^{s} \frac{f_{i}\left(x_{k}\right)}{f_{j}\left(x_{k}\right)} .
$$


Теперь из (3), (4) и определения м.о. $\widetilde{\tau}$ получаем

$$
\begin{aligned}
(m+L(\gamma)+1) \gamma & \geqslant \sum_{s=m}^{m+L(\gamma)} \mathbf{E}_{m, i \Rightarrow j}\left(\mathbb{I}(\widetilde{\tau}=s) \exp \left\{-\ln \frac{d \mathbf{P}_{m, i \Rightarrow j}}{d \mathbf{P}_{\infty, i}}\left(X_{1}^{s}\right)\right\}\right) \\
& =\sum_{s=m}^{m+L(\gamma)} \mathbf{E}_{m, i \Rightarrow j}\left(\mathbb{I}(\widetilde{\tau}=s) \exp \left\{-\sum_{k=m}^{s} \ln \frac{f_{j}\left(x_{k}\right)}{f_{i}\left(x_{k}\right)}\right\}\right) \\
& =\mathbf{E}_{m, i \Rightarrow j}\left(\exp \left\{-\sum_{k=m}^{\tilde{\tau}} \ln \frac{f_{j}\left(x_{k}\right)}{f_{i}\left(x_{k}\right)}\right\}\right) .
\end{aligned}
$$

По неравенству Иенсена получаем из (5)

$$
(m+L(\gamma)+1) \gamma \geqslant \exp \left\{-\mathbf{E}_{m, i \Rightarrow j}\left(\sum_{k=m}^{\tilde{\tau}} \ln \frac{f_{j}\left(x_{k}\right)}{f_{i}\left(x_{k}\right)}\right)\right\} .
$$

Поскольку м.о. $\tau$ - вальдовская случайная величина, можно воспользоваться обобщенным тождеством Вальда (см. [8, с. 98]). Тогда из (6) получаем

$$
(m+L(\gamma)+1) \gamma \geqslant \exp \left\{-\mathbf{E}_{m, i \Rightarrow j}(\widetilde{\tau}-m+1) \rho\left(f_{j}, f_{i}\right)\right\} .
$$

Воспользуемся теперь соотношением $\widetilde{\tau}-m+1 \leqslant(\tau-m)^{+}+1$. Тогда из (7) находим, что

$$
(m+L(\gamma)+1) \gamma \geqslant \exp \left\{-\mathbf{E}_{m, i \Rightarrow j}\left((\tau-m)^{+}+1\right) \rho\left(f_{j}, f_{i}\right)\right\} .
$$

Принимая во внимание, что м.о. $\tau$ невырожден, из (8) получаем

$$
\frac{\mathbf{E}_{m, i \Rightarrow j}(\tau-m)^{+}}{|\ln \gamma|} \geqslant \rho^{-1}\left(f_{j}, f_{i}\right)\left[1-\frac{\ln (m+L(\gamma)+1)}{|\ln \gamma|}-\frac{\rho\left(f_{j}, f_{i}\right)}{|\ln \gamma|}\right] .
$$

Учитывая, что

$$
\begin{aligned}
\mathbf{E}_{m, i \Rightarrow j}(\tau-m \mid \tau \geqslant m) & =\frac{\mathbf{E}_{m, i \Rightarrow j}(\tau-m)^{+}}{\mathbf{P}_{m, i \Rightarrow j}(\tau \geqslant m)}=\frac{\mathbf{E}_{m, i \Rightarrow j}(\tau-m)^{+}}{1-\mathbf{P}_{m, i \Rightarrow j}(\tau<m)} \\
& =\frac{\mathbf{E}_{m, i \Rightarrow j}(\tau-m)^{+}}{\mathbf{P}_{\infty, i}(\tau \geqslant m)},
\end{aligned}
$$

из последнего неравенства получаем утверждение теоремы (напомним, что $\left.L\left(\tau_{C}, r^{*}, i\right)=L(\gamma)\right)$.

3 а м е ч а н и е. В современной литературе (в частности, цитировавшейся выше) известны асимптотические границы для критерия качества детектирования (отличного от рассматриваемого выше) не только первого, но и более высоких порядков. Однако, на наш взгляд, асимптотические формулы представляют скорее теоретический, нежели 
практический интерес, поскольку для остаточных членов в таких формулах (типа $o(\cdot))$ не приводятся количественные оценки, а это не позволяет указать, когда именно асимптотические формулы начинают «работать». В то же время неасимптотическая нижняя гранииа в теореме 1 может позволить явно оценивать качество конкретного метода. Действительно, рассмотрим конкретный метод детектирования с решающей функцией $d(C, n)$ и переключением вида $0 \Rightarrow 1$, м.о. которого невырожден и имеет конечное математическое ожидание. Положим $p_{k}=\mathbf{P}_{\infty}\{d(C, k)=1\}$, $q_{k}=1-p_{k}, \gamma \stackrel{\text { def }}{=} \max _{k} p_{k}$. Если известна оценка хвоста распределения $\varphi(L) \geqslant \mathbf{P}_{\infty}\left\{\tau_{C} \geqslant L\right\}$, то нижняя граница в теореме 1 имеет вид

$$
\frac{\rho^{-1}\left(f_{1}, f_{0}\right)}{\varphi(m)}\left[1-\frac{\ln \left(m+1+\varphi^{-1}(\gamma)\right)}{|\ln \gamma|}-\frac{\rho\left(f_{1}, f_{0}\right)}{|\ln \gamma|}\right]
$$

(вместо $\varphi(m)$ можно использовать оценку $\mathbf{P}_{\infty}\left\{\tau_{C} \geqslant m\right\} \leqslant \min _{k<m} q_{k}$ ).

Нижняя граница критерия представляется в виде явной функиии от $n a p b l(m, C)$. Это обстоятельство позволяет анализировать любой конкретный метод детектирования из рассматриваемого класса и выбрать рациональное значение «большого параметра» $C$. Таким образом, введение в рассмотрение процесса $d(C, n)$ (по-видимому, впервые предложенное в наших работах) представляется целесообразным.

4. Вспомогательные результаты. Приведем некоторые вспомогательные результаты, доказательства которых (в более общей ситуации зависимых наблюдений) можно получить, используя $[7$, с. 229 и c. 261] (см. также [9]).

Пусть $i \neq j$. Положим $S_{r}=\sum_{u=1}^{r} \ln \left(f_{j}\left(x_{u}\right) / f_{i}\left(x_{u}\right)\right)$ и рассмотрим последовательности

$$
u_{n}=S_{n}-\min _{1 \leqslant r \leqslant n} S_{r}, \quad v_{n}=\max _{1 \leqslant r \leqslant n} S_{r}-S_{n} .
$$

Эти последовательности соответствуют классическому методу CUSUM: $u_{n}$ детектирует переключение с плотности $f_{i}$ на плотность $f_{j}$ (т.е. «разладку» типа $i \Rightarrow j$ ), а $v_{n}$ детектирует переключение с плотности $f_{j}$ на плотность $f_{i}$ (т.е. «разладку» типа $j \Rightarrow i$ ). Положим

$$
\tau_{i \Rightarrow j}^{\mathrm{CS}}(C)=\inf \left\{n: u_{n}>C\right\}, \quad \tau_{j \Rightarrow i}^{\mathrm{CS}}(C)=\inf \left\{n: v_{n}>C\right\} .
$$

Лемма 2. Пусть $\int \ln \left(f_{j}(x) / f_{i}(x)\right) f_{s}(x) d x<0, \int \ln \left(f_{j}(x) / f_{i}(x)\right) \times$ $f_{k}(x) d x>0, s, k \in I$. Тогда для любого $m>0$ справедливы соотношения:

$$
\begin{array}{ll}
\lim _{C \rightarrow \infty} \frac{\left(\tau_{i \Rightarrow j}^{\mathrm{CS}}(C)-m\right)^{+}}{C}=\left(\int \ln \left(\frac{f_{j}(x)}{f_{i}(x)}\right) f_{k}(x) d x\right)^{-1} & \mathbf{P}_{m, s \Rightarrow k}-n . \mu . \\
\lim _{C \rightarrow \infty} \frac{\left(\tau_{j \Rightarrow i}^{\mathrm{CS}}(C)-m\right)^{+}}{C}=\left(\int \ln \left(\frac{f_{i}(x)}{f_{j}(x)}\right) f_{s}(x) d x\right)^{-1} & \mathbf{P}_{m, k \Rightarrow s}-n . \mu .
\end{array}
$$


Положим

$$
\begin{aligned}
& \alpha_{s}^{\mathrm{CS}}(C) \stackrel{\text { def }}{=} \sup _{n} \mathbf{P}_{\infty, s}\left\{\max _{1 \leqslant r \leqslant n} \sum_{u=r}^{n} \ln \frac{f_{j}\left(x_{u}\right)}{f_{i}\left(x_{u}\right)}>C\right\}, \\
& \alpha_{k}^{\mathrm{CS}}(C) \stackrel{\text { def }}{=} \sup _{n} \mathbf{P}_{\infty, k}\left\{\max _{1 \leqslant r \leqslant n} \sum_{u=r}^{n} \ln \frac{f_{i}\left(x_{u}\right)}{f_{j}\left(x_{u}\right)}>C\right\} .
\end{aligned}
$$

Лемма 3. В условиях леммь 2 справедливьг равенства

$$
\lim _{C \rightarrow \infty} \frac{\left|\ln \alpha_{s}^{\mathrm{CS}}(C)\right|}{C}=t^{*}(j, i, s), \quad \lim _{C \rightarrow \infty} \frac{\left|\ln \alpha_{k}^{\mathrm{CS}}(C)\right|}{C}=t^{*}(i, j, k),
$$

где $t^{*}(j, i, s), t^{*}(i, j, k)$ были определень в условии (iii).

Из этих лемм и теоремы 1 непосредственно получаем, что верен следующий результат.

Теорема 2. Метод CUSUM с м.о. $\tau_{i \Rightarrow j}^{\mathrm{CS}}(C)$ является асимптотически оптимальным методом в задаче о «разладке» типа $i \Rightarrow j$. Меmод CUSUM с м.о. $\tau_{j \Rightarrow i}^{\mathrm{CS}}(C)$ является асимптотически оптимальнылм методом в задаче о «разладке» типа $j \Rightarrow i$.

5. Асимптотически оптимальные методы. В этом пункте мы рассмотрим неопределенную «разладку» на два состояния и один из вариантов неопределенной «разладки» на три состояния, после чего дадим необходимые пояснения для общего случая.

А. Два состояния. Перейдем к рассмотрению неопределенной «разладки» на два состояния (т.е. когда плотность наблюдений принимает значения $f_{1}(x)$ и $f_{2}(x)$, но априори неизвестно, какая из этих двух плотностей была до момента «разладки», а какая после). ности

Положим $S_{k}=\sum_{i=1}^{k} \ln \left(f_{2}\left(x_{i}\right) / f_{1}\left(x_{i}\right)\right)$ и рассмотрим последователь-

$$
U_{n}=S_{n}-\min _{1 \leqslant k \leqslant n} S_{k}, \quad V_{n}=\max _{1 \leqslant k \leqslant n} S_{k}-S_{n}
$$

Положим $Z_{n}=\min \left(U_{n}, V_{n}\right)$ и введем м.о. $T=\min \left\{n: Z_{n}>C\right\}$. Если ввести м.о.

$$
\tau_{1 \Rightarrow 2}=\min \left\{n: U_{n}>C\right\}, \quad \tau_{2 \Rightarrow 1}=\min \left\{n: V_{n}>C\right\}
$$

то легко видеть, что $T=\max \left(\tau_{1 \Rightarrow 2}, \tau_{2 \Rightarrow 1}\right)$.

Теорема 3. Для любого $1>\varepsilon>0$ существуют $\mathbb{C}(\varepsilon)>0 u$ $\mathbb{F}(C)>0$ такие, что если $C>\mathbb{C}(\varepsilon)$, то для любого фиксированного $m>\mathbb{F}(C)$ с вероятностью больше $(1-\varepsilon)$ м.о. $T$ является асимптотически оптимальньм в задаче о неопределенной «разладке» на два состояния. 
Д о к а з а т е л ь с т в о. Пусть «разладка» (в момент времени $m>0)$ имеет вид $1 \Rightarrow 2$. Тогда последовательность $S_{n}$ до момента $m$ имеет отрицательный снос, а после момента $m$ положительный снос, и поэтому $U_{n}$ ведет себя так же, как и в классическом методе CUSUM, а м.о. $\tau_{1 \Rightarrow 2}$ совпадает с м.о. CUSUM.

Будем считать, что момент «разладки» $m$ достаточно велик (соответствующая оценка будет приведена ниже). Зафиксируем $1>\varepsilon>0$. Последовательность $V_{n}$ в рассматриваемой ситуации до момента $m$ ведет себя так же, как и в классическом методе CUSUM, но при «разладке» вида $2 \Rightarrow 1$, которая произошла в начальный момент времени (иными словами, $\mathbf{P}_{m, 1 \Rightarrow 2}\left(\tau_{2 \Rightarrow 1} \in A\right)=\mathbf{P}_{0,2 \Rightarrow 1}\left(\tau_{2 \Rightarrow 1} \in A\right)$ для любого множества $A$ из $\sigma$-алгебры $\left.\mathscr{F}_{k}, k<m\right)$. Но, в силу леммы 2 , имеем при $C>A(\varepsilon)$

$$
\mathbf{P}_{0,2 \Rightarrow 1}\left\{\left(\rho^{-1}\left(f_{1}, f_{2}\right)-\varepsilon\right) C \leqslant \tau_{2 \Rightarrow 1} \leqslant\left(\rho^{-1}\left(f_{1}, f_{2}\right)+\varepsilon\right) C\right\} \geqslant 1-\frac{\varepsilon}{2} .
$$

Положим $\mathbf{P}=\mathbf{P}_{m, 1 \Rightarrow 2}\left\{\tau_{1 \Rightarrow 2} \geqslant m\right\}$ и оценим эту вероятность. Имеем

$$
\begin{aligned}
\mathbf{P} & \geqslant \mathbf{P}_{m, 1 \Rightarrow 2}\left\{\mathbb{D}_{1 \Rightarrow 2}(C, 1)=1, \mathbb{D}_{1 \Rightarrow 2}(C, 2)=1, \ldots, \mathbb{D}_{1 \Rightarrow 2}(C, m-1)=1\right\} \\
& \geqslant \mathbf{P}_{m, 1 \Rightarrow 2}\left\{\mathbb{D}_{1 \Rightarrow 2}(C, m-1)=1\right\}-\mathbf{P}_{m, 1 \Rightarrow 2}\left(\bigcup_{s=1}^{m-2}\left\{\mathbb{D}_{1 \Rightarrow 2}(C, s)=2\right\}\right)
\end{aligned}
$$

(здесь мы воспользовались элементарным неравенством $\mathbf{P}(A B) \geqslant \mathbf{P}(A)-$ $\mathbf{P}(\Omega \backslash B))$.

В силу предположения (iii) функция $\varkappa(t)=\ln \int\left(f_{2}(x) / f_{1}(x)\right)^{t} f_{1}(x) d x$ имеет единственный положительный корень $t^{*}=1$. Поэтому из леммы 3 следует, что при $C>B(\varepsilon)$ вероятность ложного решения допускает оценку

$$
\mathbf{P}_{m, 1 \Rightarrow 2}\left\{\mathbb{D}_{1 \Rightarrow 2}(C, k)=2\right\}=\mathbf{P}_{\infty, 1}\left\{\mathbb{D}_{1 \Rightarrow 2}(C, k)=2\right\} \leqslant \exp (-C(1-\varepsilon))
$$

для любого $k<m$. Из (10) и (11) получаем, что при $C>B(\varepsilon)$

$$
\mathbf{P} \geqslant 1-(m-1) \exp (-C(1-\varepsilon)) .
$$

Положим $\mathbb{A}(\varepsilon)=\max (A(\varepsilon), B(\varepsilon))$.

Найдем число $\mathbb{K}(\varepsilon)$ из условия

$$
\max _{x \geqslant \mathbb{K}(\varepsilon)}\left[\left(\rho^{-1}\left(f_{1}, f_{2}\right)+1\right) x-1\right] \exp (-x(1-\varepsilon)) \leqslant \frac{\varepsilon}{2}
$$

и положим $\mathbb{D}_{0}(\varepsilon)=\max (\mathbb{A}(\varepsilon), \mathbb{K}(\varepsilon))$. Тогда, если $C>\mathbb{D}_{0}(\varepsilon)$, то для любого фиксированного $m>\left[\rho^{-1}\left(f_{1}, f_{2}\right)+1\right] C \stackrel{\text { def }}{=} \mathbb{F}_{0}(C)$ из $(9),(12)$ и $(13)$ следует, что $T=\tau_{1 \Rightarrow 2}$ с вероятностью большей, чем чем $1-\varepsilon$. Тем самым утверждение теоремы для этого варианта «разладки» доказано. 
Проводя аналогичные рассуждения в случае «разладки» вида $2 \Rightarrow 1$, получаем, что тогда $T=\tau_{2 \Rightarrow 1}$ с вероятностью большей, чем $1-\varepsilon$, для любого фиксированного $m>\left(\rho^{-1}\left(f_{2}, f_{1}\right)+1\right) C \stackrel{\text { def }}{=} \mathbb{F}_{1}(C)$, если $C>\mathbb{D}_{1}(\varepsilon)$. Так как м.о. $\tau_{2 \Rightarrow 1}$ является асимптотически оптимальным при «разладке» вида $2 \Rightarrow 1$, то отсюда получаем, что утверждение теоремы верно и в этом случае. Остается положить $\mathbb{C}(\varepsilon)=\max \left(\mathbb{D}_{0}(\varepsilon), \mathbb{D}_{1}(\varepsilon)\right)$ и $\mathbb{F}(C)=\max \left(\mathbb{F}_{0}(C), \mathbb{F}_{1}(C)\right)$.

В. Три состояния. Рассмотрим теперь ситуацию, когда список возможных плотностей состоит из трех функций $f_{1}(x), f_{2}(x), f_{3}(x)$, а разладка заключается в смене любой из этих функций на любую другую. Алгоритм обнаружения в данном случае зависит от комбинации знаков чисел

$$
F=\mathbf{E}_{3} \ln \frac{f_{2}(x)}{f_{1}(x)}, \quad G=\mathbf{E}_{2} \ln \frac{f_{3}(x)}{f_{1}(x)}, \quad H=\mathbf{E}_{1} \ln \frac{f_{3}(x)}{f_{2}(x)} .
$$

Мы подробно рассмотрим случай

$$
F>0, \quad G>0, \quad H<0
$$

и дадим необходимые пояснения для всех других вариантов.

Рассмотрим статистики

$$
\begin{aligned}
U_{1}(n) & =\min _{j=2,3} \max _{1 \leqslant k \leqslant n} \sum_{s=k}^{n} \ln \frac{f_{1}\left(x_{s}\right)}{f_{j}\left(x_{s}\right)}, & U_{2}(n) & =\max _{j=2,3} \max _{1 \leqslant k \leqslant n} \sum_{s=k}^{n} \ln \frac{f_{j}\left(x_{s}\right)}{f_{1}\left(x_{s}\right)}, \\
V_{1}(n) & =\max _{1 \leqslant k \leqslant n} \sum_{s=k}^{n} \ln \frac{f_{2}\left(x_{s}\right)}{f_{3}\left(x_{s}\right)}, & V_{2}(n) & =\max _{1 \leqslant k \leqslant n} \sum_{s=k}^{n} \ln \frac{f_{3}\left(x_{s}\right)}{f_{2}\left(x_{s}\right)}
\end{aligned}
$$

и моменты остановки

$$
\begin{array}{rll}
S=\inf \left\{n: U_{1}(n)>C_{1}\right\}, & T=\inf \left\{n: U_{2}(n)>C_{2}\right\}, & \tau_{1}=\max (T, S), \\
R=\inf \left\{n: V_{1}(n)>C_{1}\right\}, & Z=\inf \left\{n: V_{2}(n)>C_{2}\right\}, & \tau_{2}=\max (R, Z), \\
\tau=\min \left(\tau_{1}, \tau_{2}\right) . & &
\end{array}
$$

Таким образом, алгоритм детектирования определяется двумя порогами, т.е. «большой параметр» $C=\left(C_{1}, C_{2}\right)$ оказывается векторным.

Поясним принцип формирования этих статистик и моментов остановки. Статистика $U_{2}(n)$ есть минимаксный вариант метода CUSUM (см. [6]), «настроенный» на переключение вида $f_{1} \Rightarrow\left(f_{2}, f_{3}\right)$, т.е. на переключение плотности $f_{1}$ на одну из (априори неизвестных) плотностей $f_{2}, f_{3}$. Иными словами, в этой ситуации до момента «разладки» имеет место простая гипотеза $f=f_{1}$, а после момента «разладки» сложная гипотеза $f=f_{2}$ или $f=f_{3}$. Действительно, условие (14) гарантирует, что до «разладки» такого вида среднее значение суммы 
$\sum_{s=k}^{n} \ln \left(f_{j}\left(x_{s}\right) / f_{1}\left(x_{s}\right)\right)$ отрицательно, а после - положительно при любой из плотностей $f_{j}=f_{2}, f_{j}=f_{3}$. Как показано в нашей работе [6], в этой ситуации м.о. $T$ является асимтотически оптимальным.

Статистика $U_{1}(n)$ есть минимаксный вариант метода CUSUM, «настроенный» на переключение вида $\left(f_{2}, f_{3}\right) \Rightarrow f_{1}$, и из той же работы [6] следует, что в этом случае м.о. $S$ является асимптотически оптимальным. Момент остановки $\tau_{1}$ позволяет «отличить» вариант $f_{1} \Rightarrow\left(f_{2}, f_{3}\right)$ от варианта $\left(f_{2}, f_{3}\right) \Rightarrow f_{1}$.

Статистика $V_{1}(n)\left(V_{2}(n)\right)$ есть вариант метода CUSUM, «настроенный» на переключение вида $f_{3} \Rightarrow f_{2}\left(f_{2} \Rightarrow f_{3}\right)$ и, как следует из теоремы 2, м.о. $R(Z)$ является асимптотически оптимальным для этого переключения. Момент остановки $\tau_{2}$ «отличает» ситуацию $f_{2} \Rightarrow f_{3}$ от ситуации $f_{3} \Rightarrow f_{2}$.

Наконец, м.о. $\tau$ позволяет детектировать любое возможное переключение.

Рассмотрим подробнее один из возникающих здесь вариантов, пользуясь рассуждениями того же типа, что и при доказательстве теоремы 3.

Переключение $f_{1} \Rightarrow f_{2}$. Будем предполагать, что момент «разладки» $m$ достаточно велик (соответствующая оценка дается ниже). Как уже отмечалось, асимптотически оптимальным (если априори известно, что переключение имеет вид $1 \Rightarrow(2,3))$, является м.о. $T$. Соответствующая решающая функция $\mathbb{D}^{\mathrm{MCS}}\left(C_{2}, n\right)$ порождается статистикой $U_{2}(n)$, причем $\mathbb{D}^{\mathrm{MCS}}\left(C_{2}, n\right)=1$, если $U_{2}(n) \leqslant C_{2}$ (нет «разладки»), $\mathbb{D}^{\mathrm{MCS}}\left(C_{2}, n\right)=2$ («разладка» типа $1 \Rightarrow 2$ ), если $\max _{1 \leqslant k \leqslant n} \sum_{s=k}^{n} \ln \left(f_{2}\left(x_{s}\right) / f_{1}\left(x_{s}\right)\right)>C_{2}$, и $\mathbb{D}^{\mathrm{MCS}}\left(C_{2}, n\right)=3$ («разладка» типа $1 \Rightarrow 3)$, если $\max _{1 \leqslant k \leqslant n} \sum_{s=k}^{n} \ln \left(f_{3}\left(x_{s}\right) / f_{1}\left(x_{s}\right)\right)>C_{2}$.

Статистика $U_{1}(n)$ в этой ситуации представляет собой минимаксный вариант метода CUSUM, «настроенный» на переключение вида $\left(f_{2}, f_{3}\right) \Rightarrow f_{1}$ (т.е., в данном случае, на «разладку» вида $2 \Rightarrow 1$ ) в начальный момент (ср. доказательство теоремы 3 ). Положим $\mathbb{R}=$ $\max \left(\rho^{-1}\left(f_{1}, f_{2}\right), \rho^{-1}\left(f_{1}, f_{3}\right)\right)$. Из [6] можно извлечь, что если $C_{1}>A(\varepsilon)$, то

$$
\mathbf{P}_{0,2 \Rightarrow 1}\left\{(\mathbb{R}-\varepsilon) C_{1} \leqslant S \leqslant(\mathbb{R}+\varepsilon) C_{1}\right\} \geqslant 1-\frac{\varepsilon}{4} .
$$

Статистика $V_{1}(n)$ ведет себя как CUSUM с «разладкой» вида $2 \Rightarrow 1$ в начальный момент (так как математическое ожидание соответствующей суммы положительно в силу условия (14)) и поэтому при $C_{1}>D(\varepsilon)$ в силу леммы 2

$$
\begin{aligned}
\mathbf{P}_{0,2 \Rightarrow 1}\{ & \left(\left[\mathbf{E}_{1} \ln \frac{f_{2}(x)}{f_{3}(x)}\right]^{-1}-\varepsilon\right) C_{1} \leqslant R \\
& \left.\leqslant\left(\left[\mathbf{E}_{1} \ln \frac{f_{2}(x)}{f_{3}(x)}\right]^{-1}+\varepsilon\right) C_{1}\right\} \geqslant 1-\frac{\varepsilon}{4} .
\end{aligned}
$$


Выберем $C_{1}>\max (A(\varepsilon), D(\varepsilon)) \stackrel{\text { def }}{=} \mathbb{C}(\varepsilon)$ и зафиксируем

$$
m>C_{1} \max \left(\mathbb{R}+1,\left[\mathbf{E}_{1} \ln \frac{f_{2}(x)}{f_{3}(x)}\right]^{-1}+1\right) \stackrel{\text { def }}{=} \mathbb{F}\left(C_{1}\right) .
$$

Приведем теперь соотношение, которое можно извлечь из [6] и которое в рассматриваемой ситуации имеет следующий вид: для любого фиксированного $m$ имеет место равенство

$$
\begin{aligned}
\lim _{C_{2} \rightarrow \infty} \frac{(T-m)^{+}}{C_{2}} & =\min \left(\rho^{-1}\left(f_{2}, f_{1}\right),\left(\mathbf{E}_{2} \ln \frac{f_{3}(x)}{f_{1}(x)}\right)^{-1}\right) \\
& =\rho^{-1}\left(f_{2}, f_{1}\right) \quad \mathbf{P}_{m, 1 \Rightarrow 2} \text {-П.н. }
\end{aligned}
$$

Отсюда следует, что при любом фиксированном $m$

$$
\mathbf{P}_{m, 1 \Rightarrow 2}\left\{m+\left(\rho^{-1}\left(f_{2}, f_{1}\right)-\varepsilon\right) C_{2} \leqslant T \leqslant m+\left(\rho^{-1}\left(f_{2}, f_{1}\right)+\varepsilon\right) C_{2}\right\} \geqslant 1-\frac{\varepsilon}{4},
$$

если $C_{2}>F(m, \varepsilon)$.

Если так выбран второй порог, то в силу условия на $m$ и выбора $C_{1}$ получаем, что

$$
\mathbf{P}_{m, 1 \Rightarrow 2}\{T>S\} \geqslant 1-\frac{\varepsilon}{2} .
$$

Статистика $V_{2}(n)$ ведет себя как CUSUM, когда «разладка» отсутствует (так как математическое ожидание соответствующей суммы не меняет отрицательный знак). Пусть $t_{1}^{*}-$ положительный корень уравнения $\ln \mathbf{E}_{1}\left(f_{3}(x) / f_{2}(x)\right)^{t}=0$ (положительным корнем уравнения $\ln \mathbf{E}_{2}\left(f_{3}(x) / f_{2}(x)\right)^{t}=0$ является 1$)$.

Тогда из леммы 3 и рассуждений, аналогичных использованным при выводе соотношений (10)-(12), получаем, что при $C_{2}>H(\varepsilon), k \geqslant 0$

$\mathbf{P}_{m, 1 \Rightarrow 2}\{Z \geqslant m+k\} \geqslant 1-(m-1) \exp \left(-t_{1}^{*} C_{2}(1-\varepsilon)\right)-(k-1) \exp \left(-C_{2}(1-\varepsilon)\right)$.

Выберем $L(m, \varepsilon)$ из условия

$$
\begin{aligned}
& \max _{x \geqslant L(m, \varepsilon)}\left[(m-1) \exp \left(-x t_{1}^{*}(1-\varepsilon)\right)\right. \\
& \left.\quad+\left(\left(\rho^{-1}\left(f_{2}, f_{1}\right)+1\right) x-1\right) \exp (-x(1-\varepsilon))\right] \leqslant \frac{\varepsilon}{4}
\end{aligned}
$$

и положим $\mathbb{G}(m, \varepsilon)=\max (H(\varepsilon), F(m, \varepsilon), L(m, \varepsilon))$. Тогда, если $C_{1}>\mathbb{C}(\varepsilon)$, $m>\mathbb{F}\left(C_{1}\right)$ фиксировано и $C_{2}>\mathbb{G}(m, \varepsilon) \stackrel{\text { def }}{=} \Phi\left(C_{1}, \varepsilon\right)$, то из (15)-(20) следует, что

$$
\mathbf{P}_{m, 1 \Rightarrow 2}\{\tau=T\} \geqslant 1-\varepsilon .
$$

Таким образом, $\tau=T$ с большой вероятностью, т.е. алгоритм с большой вероятностью обнаруживает эту «разладку» так же, как асимптотически оптимальный метод. 
Аналогичного рода рассуждения проводятся и при всех других вариантах переключения.

Таким образом, установлена следующая теорема.

Теорема 4. Пусть выполнены условия (14). Тогда существуют функиия $\mathbb{F}(\cdot)>0$ и для любого $1>\varepsilon>0$ функиии $\mathbb{C}(\varepsilon)>0, \mathbb{G}(\cdot, \varepsilon)>0$ такие, что если $C_{1}>\mathbb{C}(\varepsilon)$, то для любого фиксированного $m>\mathbb{F}\left(C_{1}\right)$ при выборе $C_{2}>\mathbb{G}(m, \varepsilon)$ с вероятностью больше, чем $1-\varepsilon$ описанный метод обнаружения любого из возможных переключений является асимптотически оптимальным.

Дадим теперь пояснения для общей ситуации конечного списка плотностей (в частности, из этого описания можно извлечь вид алгоритма обнаружения и для всех комбинаций знаков в (14)).

Список плотностей $I$ разбивается на два подмножества $A$ и $B$ так, чтобы выполнялось условие

$$
\min _{s \in A} \min _{i \in A, j \in B} \mathbf{E}_{s} \ln \frac{f_{i}(x)}{f_{j}(x)}>0>\max _{s \in B} \max _{i \in A, j \in B} \mathbf{E}_{s} \ln \frac{f_{i}(x)}{f_{j}(x)} .
$$

Условие (21) дает возможность ввести в рассмотрение «разладку» типа $A \Rightarrow B$ или $B \Rightarrow A$, т.е. «разладку» при сложных гипотезах как до момента изменения $\left(f_{i}, i \in A\right.$ или $f_{i}, i \in B$ ), так и после (выше при условии (14) выбирается $A=\{1\}, B=\{2,3\})$. Для детектирования такого момента изменения надо сформировать статистики минимаксного CUSUM (ср. $U_{1}(n), U_{2}(n)$ выше). Максимум из соответствующих м.о. (ср. $\tau_{1}$ выше) позволяет отличить вариант $A \Rightarrow B$ от варианта $B \Rightarrow A$. Далее, каждое из подмножеств $A$ и $B$ следует разделить на два подмножества по тому же принципу, который был использован в (21) (выше подмножество $B$ делится на подмножества $\{2\}$ и $\{3\}$ ), и вновь сформировать статистики минимаксного CUSUM, которые позволили бы отличать вновь образованные подмножества друг от друга (выше это были статистики $V_{1}(n)$ и $\left.V_{2}(n)\right)$. При этом выбор направления переключения внутри подмножеств $A$ и $B$ определяется максимумом соответствующих м.о. (ср. $\tau_{2}$ выше). Эту процедуру следует продолжать до тех пор, пока весь список плотностей не разделится на подмножества, каждое из которых состоит только из одного элемента. Момент остановки, который будет детектировать произвольное переключение, будет определяться минимумом всех ранее построенных м.о. (ср. выше м.о. $\tau$ ).

Если момент «разладки» достаточно далек от начала наблюдений, то при правильном подборе порогов описанный алгоритм с большой вероятностью будет совпадать с асимптотически оптимальным алгоритмом при произвольном переключении плотностей из заданного списка.

6. Субоптимальный непараметрический метод детектирования при трех состояниях. В этом пункте мы рассмотрим метод 
детектирования, который, вообще говоря, не является асимптотически оптимальным (если не делать специальных предположений о распределениях), но зато не требует знания плотностей и может быть практически удобным.

Предположим, что математическое ожидание наблюдаемой последовательности $X=\{x(n)\}$ может принимать три известных значения $m_{1}<m_{2}<m_{3}$, а (неопределенная) «разладка» состоит в изменении математического ожидания с любого из этих значений на любое другое (еще раз подчеркнем, что мы не предполагаем априорньх знаний о том, каким было математическое ожидание до «разладки», а каким после). Мы будем использовать непараметрический вариант метода CUSUM, ориентированный на обнаружение «разладки» по математическому ожиданию, который описан в [7] для более общей ситуации зависимых наблюдений (заметим, что всегда можно свести задачу обнаружения момента изменения любой вероятностной характеристики к задаче обнаружения математического ожидания для некоторой вспомогательной последовательности, построенной по исходной - это было отмечено в наших работах 70-х годов).

Положим $A=\left(m_{1}+m_{2}\right) / 2, B=\left(m_{2}+m_{3}\right) / 2$ и будем рассматривать последовательности

$$
y_{1}(n)=x(n)-A, \quad y_{2}(n)=x(n)-B
$$

Введем следующие статистики:

$$
\begin{array}{ll}
u_{1}(n)=\left(u_{1}(n-1)+y_{1}(n)\right)^{+}, & u_{1}(0)=0 \\
v_{1}(n)=\left(v_{1}(n-1)-y_{1}(n)\right)^{+}, & v_{1}(0)=0
\end{array}
$$

и

$$
\begin{array}{ll}
u_{2}(n)=\left(u_{2}(n-1)+y_{2}(n)\right)^{+}, & u_{2}(0)=0 \\
v_{2}(n)=\left(v_{2}(n-1)-y_{2}(n)\right)^{+}, & v_{2}(0)=0 .
\end{array}
$$

Статистика $u_{1}(n)\left(v_{1}(n)\right)$ есть непараметрический вариант метода CUSUM, настроенного на изменение знака математического ожидания последовательности $\left\{y_{1}(n)\right\}$ с минуса на плюс (с плюса на минус). То же самое относится и к статистике $u_{2}(n)\left(v_{2}(n)\right)$ применительно к последовательности $\left\{y_{2}(n)\right\}$.

Введем моменты остановки

$$
\begin{aligned}
T_{1} & =\inf \left\{n: u_{1}(n)>C_{1}\right\}, \quad S_{1}=\inf \left\{n: v_{1}(n)>C_{2}\right\} \\
T_{2} & =\inf \left\{n: u_{2}(n)>C_{1}\right\}, \quad S_{2}=\inf \left\{n: v_{2}(n)>C_{2}\right\} \\
\tau_{1} & =\max \left(T_{1}, S_{1}\right), \quad \tau_{2}=\max \left(T_{2}, S_{2}\right), \\
\tau & =\min \left(\tau_{1}, \tau_{2}\right) .
\end{aligned}
$$


Если порог $C=\left(C_{1}, C_{2}\right)$ достаточно велик, а момент «разладки» достаточно далек от начала наблюдений, то м.о. $\tau$ будет детектировать произвольное изменение математического ожидания в исходной последовательности.

Поясним, как работает этот алгоритм, на примере переключения математического ожидания со значения $m_{1}$ на значение $m_{2}$. При таком переключении математическое ожидание последовательности $\left\{y_{1}(n)\right\}$ меняет знак (в момент «разладки») с плюса на минус, а положительный знак математического ожидания последовательности $\left\{y_{2}(n)\right\}$ не меняется в результате «разладки». Поэтому статистики $u_{1}(n)$ и $u_{2}(n)$ ведут себя как непараметрический CUSUM с «разладкой» в начальный момент. Статистика $v_{1}(n)$ ведет себя как непараметрический CUSUM c «разладкой» типа $m_{1} \Rightarrow m_{2}$, а статистика $v_{2}(n)$ ведет себя как непараметрический CUSUM при отсутствии «разладки». С учетом этих замечаний построение м.о. $\tau$ производится по тем же соображениям, которые были описаны в п. 5 .

Описанный подход нетрудно обобщить на случай, когда математическое ожидание наблюдаемой последовательности может в результате «разладки», принимать любое значение из конечного множества.

Автор глубоко благодарен А. Н. Ширяеву за внимание и поддержку и признателен рецензенту, замечания которого позволили улучшить изложение.

\section{СПИСОК ЛИТЕРАТУРЫ}

1. Ширяев А.Н. О стохастических моделях и оптимальных методах в задачах скорейшего обнаружения. - Теория вероятн. и ее примен., 2008, т. 53, в. 3, с. 417436.

2. Lai T. L. Sequential multiple hypothesis testing and efficient fault detection-isolation in stochastic systems. - IEEE Trans. Inform. Theory, 2000, v. 46, № 2, p. 595-608.

3. Nikiforov $I$. $V$. A simple recursive algorithm for diagnosis of abrupt changes in random signals. - IEEE Trans. Inform. Theory, 2000, v. 46, № 7, p. 2740-2746.

4. Nikiforov I. V. A lower bound for the detection/isolation delay in a class of sequential tests. - IEEE Trans. Inform. Theory, 2003, v. 49, № 11, p. 3037-3047.

5. Tartakovsky A.G. Multidecision quickest change-point detection: previous achievements and open problems. - Sequential Anal., 2008, v. 27, № 2, p. 201-231.

6. Brodsky B., Darkhovsky B.. Minimax methods for multihypothesis sequential testing and change-point detection problems. - Sequential Anal., 2008, v. 27, № 2, p. 141173.

7. Brodsky B. E., Darkhovsky B.S. Non-Parametric Statistical Diagnosis: Problems and Methods. Dordrecht: Kluwer, 2000, 452 p.

8. Круглов В.М., Королев В.Ю. Предельные теоремы для случайных сумм. М.: Изд-во МГУ, 1990, 269 с.

9. Боровков A. A. Асимптотически оптимальные решения в задаче о разладке. Теория вероятн. и ее примен., 1998, т. 43, в. 4, с. 625-654.

Поступила в редакцию 26.IV.2010 